\title{
Downlink HARQ feedback for LTE- A systems with carrier aggregation
}

\author{
Fatang Chen ${ }^{1} \mathrm{Xin} \mathrm{Ni}^{1}$ \\ ${ }^{1}$ Key Laboratory of Mobile Communication Technology of Chongqing, Chongqing University of Posts and Telecommunications, \\ Chongqing 400065 \\ SmallMonkey168@163.com
}

\begin{abstract}
Carrier aggregation (CA) technology is one of key technologies in LTE-Advanced(LTE-A) system. UE can aggregate multiple component carriers (CCs) as a wider band to support the demand of higher data rates and improve the spectrum utilization. When terminal aggregates multiple downlink carrier components at the same time, LTE-A terminal needs to be feedback 20 HARQ-ACK bits at most, so that LTE-A system adds PUCCH format3.This paper presents an efficient method for feeding back large downlink HARQ information to support a variety of terminals with different bandwidth capacity.
\end{abstract}

Keyword : LTE-A ; CA ; PUCCH

\section{Introduction}

One of the key requirements for International Mobile Telecommunications Advanced(IMT-Advanced) is supporting up to $100 \mathrm{MHz}$ bandwidths specified by the International Telecommunication Union-Radio communication Sector(ITU$\mathrm{R})^{[1][2]}$.

Carrier aggregation technology $(\mathrm{CA})$ is one of the key technologies in LTE-A system, which can effectively solve the bandwidth extension problem and improve the spectrum utilization. In order to support the wider bandwidth and guarantee the accuracy of the information transmission at the same time, LTE-A puts forward with the solution project, the carrier aggregation technology combined with hybrid automatic retransmission request (HARQ). According to uplink control channel design, LTE-A system introduces the PUCCH format. $3^{[3]}$.

\section{Multi-carriers aggregation technology principle ${ }^{[4]}$}

LTE-A carrier aggregation technology can aggregate a set of multiple continuous or discrete carrier into a wider spectrum, to support the largest bandwidth of $100 \mathrm{MHZ}$, downward peak rate $1 \mathrm{Gbit} / \mathrm{s}$ and the peak uplink rate 500 $\mathrm{Mbit} / \mathrm{s}$ in LTE-A system. CA technology can meet the LTE-A large bandwidth requirement, and improve the utilization rate of spectrum fragments.

In the each component carrier UE aggregates, the frame structure is the same with LTE.LTE-A terminal can send/receive five component carriers at the same time, and each component carrier can most support 100 resources . All carriers of LTE-A is configured with the same standard of LTE carrier, and LTE terminals are able to send/receive data on the one of the component carrier. UE can support asymmetric carriers, namely the different number of the downlink and uplink aggregation component carriers.

\section{Main and Complementary Carriers Allocation mechanism}

UE aggregates multiple component carriers, including primary component carriers including uplink and downlink carriers and secondary component carriers configured by highlevel. The LTE-A protocol demands that primary component carrier must contain a pair of uplink and downlink carriers and secondary carrier component can allow only a downlink carrier. This division is because of the different role of primary and secondary component carriers, for example: the random access process initiated by terminal can be only sent in the main component carrier; the main component carrier are always in activation state, and secondary component carrier can be in activation state or idle state decided by high-level; the mobile information, security parameters will be only sent on primary carrier component.

\section{The Design of Uplink Control Channel}

The LTE-A protocol regulates that UE can only send a PUCCH information on the primary component carrier. If UE aggregates multiple downlink carrier components, every time UE needs to feedback more HARQ bits, LTE-A system adds PUCCH format 3 to support most 20 HARQ bits. To maintain backward compatibility, in LTE-A system each component carrier can keep the same structure as that of LTE system.

\section{A. Terminal determine the feedback $H A R Q$}

When UE uses PUCCH format 3 to feedback the HARQACK bits configured by high-layer, according to the configuration, number of carrier components terminal $\left(N_{\text {cell }}\right)$, uplink and downlink proportional relationship (1:m) and the transmission mode ( $\mathrm{n}$ for one code or two code), the all parameters determine total number of feedback HARQ bits

$$
\begin{aligned}
& \left(N_{A / N}^{\text {PUCCH format3 }}\right) . \\
& \quad N_{A / N}^{\text {PUCCHformat } 3}=N_{\text {cell }} \times m \times n
\end{aligned}
$$

If the proportion of up and down is 1:4 in a wireless frame, one uplink sub-frame need feedback 4 HARQ bits corresponding to downlink sub-frames. Assuming that UE converges five component carrier, and the transfer mode of each component supports two code word of flow transmission, the UE needs to feedback 40 HARQ bits. 


\section{B. The HARQ information produce process when UE adopts} the PUCCH format 3

If the number of HARQ bits exceeds 20, the first UE operates spatial logic AND of code 1 and code 2 of each down sub-frame needed in carrier component respectively, to ensure that the number of HARQ-ACK bits sent is not more than 20 .

After AND operator, if the number of HARQ -ACK bits is not more than 11 bits, UE uses data code based on single RM structure, otherwise, dual structure the RM code or PUCCH1b multiplexing feedback HARQ scheme in LTE system to ensure that the final feedback number is less than 11 .

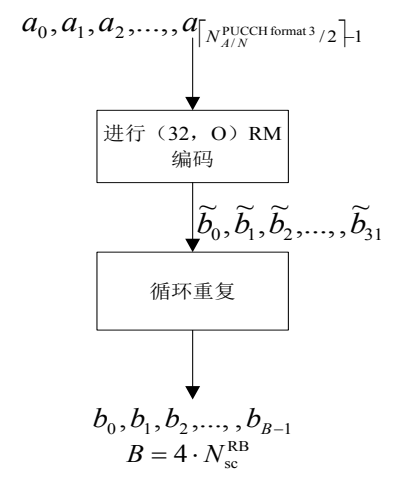

Fig1: the PUCCH format 3 single RM encoding process

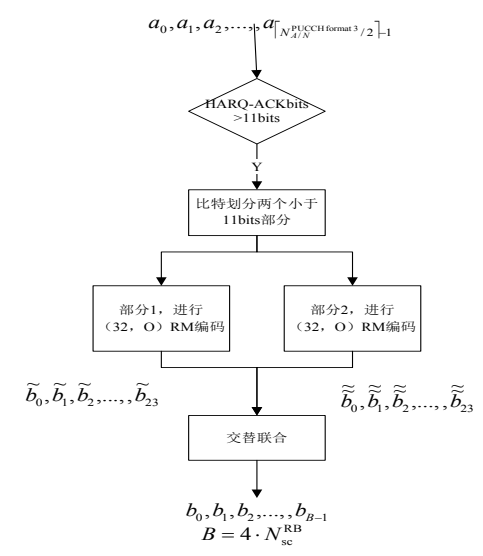

Fig2: the PUCCH format 3 double RM encoding process

Throughput is one of the important measurement standards for a system, LTE-A add new PUCCH format 3 to improve the downlink throughput. If a LTE-A system only have a downlink carrier component, UE can also use PUCCH3 to feedback HARQ bit. With uplink-downlink configuration 5 of TD-LTE, the ratio of uplink and downlink is 1:9. In order to reduce the amount of feedback HARQ bits, UE can collect only two component carriers limited by the scenario.

\section{THE SIMULATION RESULTS}

\section{A. The simulation environment}

To facilitate simulation, assume that each carrier of LTEA system keep the same configuration and frame structure in that of LTE system and use scenarios EPA5, the AWGN, and 10000 time cycles,.
Table I: the simulation parameter table

\begin{tabular}{|c|c|c|}
\hline parameters & parameters & values \\
\hline Uplink bandwidth: $25 \mathrm{RB}$ & Channel type & EPA5 \\
\hline carrier frequency $2 \mathrm{G} \mathrm{Hz}$ & doppler frequency & $5 / 300 \mathrm{~Hz}$ \\
\hline Antenna pattern $1 \mathrm{~T} 2 \mathrm{R}$ & \multicolumn{2}{|c|}{ feedback $\mathrm{A} / \mathrm{N}$ per carrier 2} \\
\hline Modulation method QPSK & noise types & AWGN \\
\hline FFTpoints & & \\
\hline
\end{tabular}

\section{B. The simulation results}

According to the requirements, the following images are obtained by MATLAB simulation.

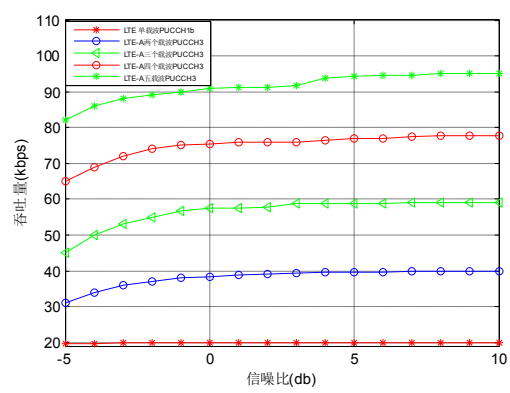

Fig 3. PUCCH3 simulation in EPA5 scenarios

Each curve in the figure is for one type of aggregation. With the improvement of the channel quality, the throughput increases gradually. When the number of aggregation carrier increases, the throughput is also increasing. But the above figures show that the throughput is not multiplied as the increase number of carriers, the reason is that the feedback information is too much, and in case of the multi-path effect and noise interference, the probability of wrong solution information goes up on the receive terminal.

\section{Conclusion}

This paper introduces CA technology and HARQ feedback in the LTE-A system with PUCCH format 3, and the produce process of $\mathrm{PUCCH}$ format 3, and analyzes the performance of HARQ feedback. Through the simulation analysis, the new HARQ solution has more throughput and improves spectrum utilization. Due to feedback large HARQ information, how to guarantee the receive terminal can solve rightly information, more reliable channel code will be further study. The frame structure of each component carrier is the same because of the simplified setup, that results in UE does not take fully LTE-A's advantage. So the different type carriers aggregated is the next research direction.

\section{References}

[1] Shen Jia SuoShiJiang. 3 GPP long term evolution (LTE) technology principle and system design [M]. Beijing: people's posts and telecommunications press, 2008

[2] 3GPP TS 36.211 v10.0: Evolved Universal Terrestrial Radio Access (E_UTRA); Physical Channel and Modulation. (release 8 ) [S]. 2009-12

[3] 3GPP TS 36.213 v10.0 Evolved Universal Terrestrial Radio Access (EUTRA) Multiplexing and channel coding (Release 9) [S]. 2009-12

[4]Wang Wentao, a LTE - under the Advanced carrier aggregation method to reduce the CM. Electronic technology application. 2012-3 